\title{
Understanding the Mechanistic Link between Bisphenol A and Cancer Stem Cells: A Cancer Prevention Perspective
}

\author{
Cassandra Winz ${ }^{1,2}$, Nanjoo Suh ${ }^{1,3}$ \\ ${ }^{1}$ Department of Chemical Biology, Ernest Mario School of Pharmacy, ${ }^{2}$ Toxicology Graduate Program, Rutgers, The State \\ University of New Jersey, Piscataway, NJ, USA, ${ }^{3}$ Rutgers Cancer Institute of New Jersey, New Brunswick, NJ, USA
}

Endocrine disruptors, such as bisphenol A (BPA), have become more frequently present in the environment as contaminants, especially in industrialized countries. Long-term effects of these environmental contaminants in humans are elusive. With their structural similarity to estrogen, many environmental contaminants including BPA, have been shown to mimic the biological functions of estrogen, potentially contributing to the development of breast cancer. It has been well established that BPA exerts estrogenic activity in animal models and in vitro systems. There is a concern for adverse effects from the exposure to BPA in regard to developmental and reproductive toxicities. However, the mechanisms by which BPA promotes breast cancer development remain unknown. Understanding the role of endocrine disruptors and their key mechanisms of action is important for public health, especially by providing a foundation for a better intervention approach in cancer prevention.

Key Words Breast cancer, Cancer stem cells, Endocrine disruptors, Estrogen receptor

\section{INTRODUCTION}

Breast cancer is one of the most commonly diagnosed cancers in women [1]. There are well established risk factors for breast cancer, such as genetic alterations, age, family history, life-style, hormone replacement therapy, and obesity [2]. According to the American Cancer Society, more than two thirds of breast cancer at the first diagnosis in women is estrogen receptor (ER)-positive [2]. Extended exposure to estrogen is epidemiologically and experimentally associated with an increased risk of developing breast cancer [1]. This appears to be due to the upregulation of genes involved in breast cell proliferation that is mediated through the estrogen receptor. Development of ER-positive breast cancer depends on estrogen for cell growth. Many environmental chemicals are endocrine disrupters that can mimic the biological functions of estrogen, potentially contributing to the initiation or progression of breast cancer.

Among known environmental estrogens, bisphenol A (BPA) is widely used in industry. The National Health and Nutrition Examination Survey (NHANES) during 2003 to 2004 indicates widespread exposure to BPA in the US population [3-5]. Since then, there is a temporal trend in decreased exposure to BPA in the United States from 2011 to 2012 [6]. However, in a recent cohort study conducted in the United States, it is concluded that higher exposure to BPA is associated with an increased risk of long-term mortality including cancer [7]. BPA has been well known to have estrogenic activity in animal models and in vitro [8-12]. There is a concern for adverse effects of the exposure to BPA in regards to developmental toxicity for fetuses, infants and children, effects on the mammary gland and early puberty in females, and reproductive toxicities [13-15]. Importantly, the health outcomes from environmental exposures are highly complex and variable.

Many studies looking at the molecular mechanisms through which BPA is thought to induce breast cancer have shown promising results. However, epidemiological studies aimed to uncover a link between BPA exposure and an increased breast cancer risk have been largely inconclusive. It is difficult to evaluate long term effects of endocrine disruptor exposure via retrospective studies, which lack specificity. Prospective cohort studies can be more specific, but they may take many years to complete. Even still, prospective studies often don't have a sample size large enough to yield significance. Due to the relatively low prevalence of breast cancer incidence within a normal population, correlation can be very hard to 
tease out. Additionally, endocrine disrupting compounds have been shown to produce differing effects depending on the time and duration of exposure, further complicating the study of the epidemiology of these compounds throughout a lifetime [16]. BPA was linked to an increased sensitivity to mammary developmental signaling in prenatal rats, suggesting a mechanism through which perturbation is dependent on the developmental time window [17]. Overall, key mechanisms of BPA in promoting breast cancer development remain largely unknown.

BPA has previously been shown to disrupt mammary gland development and breast cell morphogenesis, potentially leading to breast cell malignancies. In an MCF-12A model, low doses of BPA induced disruption of acinar structures and lumen invasion, contributing to breast cancer development [18]. It has been shown that perinatal exposure to low doses of BPA was associated with increased intraductal hyperplasia in adult female mice [19]. BPA treatment of normal mammary epithelial cells facilitated malignant cellular transformation by decreasing tubule formation and increasing the presence of spherical masses in an MCF-10F model [20]. Thus, it is important that a focus is also placed on testing the carcinogenicity of endocrine disruptors including BPA, as well as potential prevention measures against endocrine disruptor-induced cancers in animal and tissue culture models. From this, we can get a better understanding of the molecular mechanisms that perhaps contribute to this hypothesized correlation between endocrine disruptors and reproductive cancers.

\section{BPA AS AN ENVIRONIMENTAL CONTAMINANT-ENDOCRINE DISRUPTOR}

BPA is an industrial synthetic compound found in polycarbonate plastics and epoxy resins. The toxicant can leach from plastic upon heating, as well as upon exposure to mild detergents, leading to contamination of food and water supply [21]. In fact, biomonitoring the NHANES studies have consistently found detectable levels of BPA in the urine and serum of study participants $[5,22]$. As a result, BPA is widely considered to be ubiquitous.

The structural similarities between BPA and estrogen allow BPA to exert an estrogenic effect physiologically (Fig. 1). It has been postulated that the increased incidence of reproductive cancers observed over the past half century can be linked to the introduction of synthetic endocrine disruptors into the environment through industrial production of compounds like BPA and its related alternatives (also shown in Fig. 1) in common, daily-use products [23]. In fact, chronic exposure to estrogens and estrogen receptor agonists, like BPA, has been identified as a risk factor for the development of breast cancer [24-26]. Endocrine disruptors can interfere with many downstream targets of estrogen signaling, which are related to cell cycle regulation and cellular proliferation, and therefore posing a mechanism for breast cancer development [26].
Studies looking at the epidemiology of endocrine disruptor exposure as a risk factor for reproductive cancers often do not take into account the age-dependent constrictions associated with developmental endocrine systems [27]. It has been suggested that low dose exposures to endocrine disruptors during reproductive development, when the body is more sensitive to endocrine signaling, could increase cancer incidence later in life. This makes it more difficult to correlate endocrine-disruptor exposure with cancer development in retrospective epidemiological studies. Therefore, mechanistic studies on whole animal or in vitro models are useful in elucidating the involvement of BPA and other endocrine disruptors in breast cancer risk early in development.

Of particular concern, BPA-induced cancers show increased resistance against widely used chemotherapeutic agents, such as doxorubicin and cisplatin [28]. Huang et al. [29] found that low doses of BPA reversed the effects of tamoxifen-induced cell apoptosis and cell cycle regulation in MCF-7 cells through an estrogen receptor signaling pathway. Another study revealed that low doses of BPA reduced the cytotoxicity of doxorubicin, cisplatin, and vinblastine in

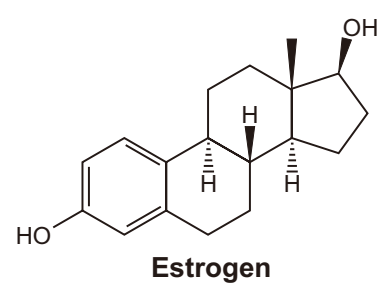<smiles>CC(C)(c1ccc(O)cc1)c1ccc(O)cc1</smiles><smiles>CCC(C)(c1ccc(O)cc1)c1ccc(Br)cc1</smiles><smiles>Oc1ccc(Cc2ccc(Br)cc2)cc1</smiles><smiles>Oc1ccc(C(c2ccc(O)cc2)(C(F)(F)F)C(F)(F)F)cc1</smiles>

BPAF<smiles>CC(c1ccccc1)(c1ccc(O)cc1)c1ccc(O)cc1</smiles>

BPAP

Figure 1. Chemical structures of estrogen and synthetic bisphenols. Alternatives of bisphenol A (BPA), bisphenol AF (BPAF, hexafluorobisphenol $A$ ), bisphenol $F$ (BPF), and bisphenol $S$ (BPS), bisphenol B (BPB), and bisphenol AP (BPAP, 4,4'-[1-Phenylethylidene] bisphenol) are also shown. 
both ER $\alpha$ positive and ER $\alpha$ negative breast cancer cell lines through increased expression of anti-apoptotic proteins [30]. A mechanistic understanding of BPA's estrogenic effects is required to elucidate the pathway through which chemotherapy resistance is conferred to breast cancer cells.

\section{MOLECULAR MECHANISIMS OF BPA IN BREAST CANCER}

\section{Established mechanisms of estrogen/ ER-mediated BPA action}

BPA's structural similarity to estrogen allows it to bind estrogen receptor $\alpha$ and $\beta$, as well as the membrane bound estrogen receptor G-protein-coupled receptor 30 (GPR30) [31,32]. In addition to its binding affinity, BPA has also been demonstrated to have intrinsic activity for ER $\alpha$. Agonistic binding of BPA to ER $\alpha$ was found to activate the same apoptotic regulation pathways as estrogen using RT-PCR and chromatin immunoprecipitation assays in an MCF-7 model [33]. Because of its ability to bind and activate ER $\alpha$, BPA is thought to modulate the expression of estrogen responsive genes, leading to the induction of downstream signaling pathways involved in cell cycle regulation and proliferation [34].

BPA was found to modulate cell cycle and apoptosis regulating proteins downstream of various estrogen signaling pathways, ultimately leading to increased cell proliferation in an MCF-7 model [35]. A unique in-vitro in-vivo model using MCF-10F cells exposed to BPA showed increased expression of genes involved in DNA repair, notably BRCA1, BRCA2, BARD1, CtIP, RAD51, and BRCC3, and decreased expression of genes involved in apoptosis, notably $P D C D 5$ and BCL2L11. These observations were comparable to estrogen exposure, exhibiting the estrogenic effects of BPA [36]. Signaling through estrogenic pathways is the primary mechanism through which BPA is suggested to induce its cancer-causing effects. However, downstream pathways ultimately leading to the initiation, proliferation, and metastasis of breast cancer through BPA induced ER $\alpha$ signaling is highly contested, and new pathways and mechanisms are constantly being suggested.

p53, a key regulator of the cell cycle that functions as the "guardian of the genome" is an important downstream signaling target of estrogen. Both BPA and $\beta$-estradiol induced p53 and ER $\alpha$ expression, as well as cellular proliferation, in cultured MCF-7 cells [37]. These findings suggest that BPA modulates expression and function of p53 through an estrogen receptor-mediated pathway. Vascular endothelial growth factor (VEGF) plays an important role in tumor angiogenesis, and increased expression of VEGF signaling is associated with cancer incidence and metastasis. BPA induced VEGF expression in a dose-dependent manner in estrogen-receptor positive MELN (derived from MCF-7 cells by stably expressing the estrogen-responsive ERE-luciferase) cell culture [38]. Additionally, VEGF has been identified as a target gene of estrogen signaling, suggesting BPA's induction of VEGF expression is perhaps ER $\alpha$-mediated [39].

STAT3 is an oncogene important to breast cancer metastasis that has been linked to the estrogen signaling pathways [40]. Zhang et al. [41] found that BPA increased cell proliferation in MCF-7 cells through upregulation of STAT3 expression. When STAT3 was silenced using RNA interference, BPA did not induce cellular proliferation, indicating a potential role of the Epidermal growth factor receptor (EGFR)-STAT3STAT3 pathway in mediating BPA's role in breast cancer [41]. Interestingly, recent studies have suggested that STAT3 signaling might induce breast cancer through an estrogen receptor independent pathway [42]. More research is required to clarify the involvement of STAT3 in estrogen receptor positive breast cancers.

The orphan receptor estrogen-related receptor gamma $(E R R \gamma)$ has also been implicated in BPA activity in breast cancer cell lines. Low doses of BPA induced cell proliferation via extracellular signal-regulated kinase 1/2/ERR $\gamma$ signaling in MCF-7 and SkBr3 cell lines. Treatment with ER $\alpha$ and G-protein-coupled estrogen receptor inhibitors had no effect on proliferation induced by BPA, while treatment with an ERR $\gamma$ inhibitor diminished the proliferative activity of BPA [43]. Yet another study suggests that BPA's estrogenic activity is conferred through the membrane bound estrogen receptor, GPR30 [44]. Environmental endocrine disruptors, including BPA and more recently bisphenols $F$ and $S$, have been suspected to play a role in increased breast cancer risk, possibly via ER-dependent mechanisms. Recent evidence suggests that increased breast cancer stem cells and epigenetic changes may contribute to cancer progression, metastasis and treatment resistance. It would be highly valuable to understand the key mechanisms by which BPA can target and enhance breast cancer stem cells and estrogen-mediated events during breast cancer development.

\section{Mechanisms of BPA modulation of cancer stem cells}

Cancer stem cells represent a subpopulation of cancer cells that possess stem-like properties and the ability for self-renewal. They are implicated in the growth and metastasis of many cancers, including breast cancer, and have been regarded with new interest as an important component of cancer initiation and relapse [45]. Of additional importance, breast cancer stem cells are thought to be the principal mediators of radiation and chemotherapy resistance [46]. For these reasons, cancer stem cells are being studied more extensively in the field of breast cancer research.

Cancer stem cells are characterized by a number of molecular factors, such as CD44 and CD24 cell surface markers, aldehyde dehydrogenase-1 (ALDH-1) expression, and in the case of breast cancer stem cells, increased mammosphere formation [45]. Other important markers involved in prolonging a stem cell phenotype include Nanog, Oct4, and Sox- 
2 [47]. The role of estrogen signaling pathways in inducing an increased breast cancer stem cell phenotype has been suggested [48]. Due to the structural and functional similarities between estrogen and BPA, newfound interest has been focused on the involvement of BPA in inducing a stem-cell phenotype to breast cancer cells.

In tissue culture, BPA induced differentiation of human embryonic stem cells into mammary epithelial cells, potentially via upregulating Nanog, Oct4 and breast cancer stem cell markers. These results indicate that BPA may contribute to the formation and maintenance of breast cancer stem cells [49]. Additionally, BPA induced Sox-2 and ALDH-1 expression in MCF-7 cells, and increased mammosphere formation in MCF-7 or patient-derived xenograft (PDX) cells. However, BPA failed to enhance the mammosphere formation in ER-negative MDA-MB-231 breast cancer cells. These findings suggest that BPA-induced manifestation of the cancer stem cell phenotype is mediated by ER. A mechanistic study found that BPA induced SOX-2 mRNA expression through cyclic AMP-responsive element binding protein (CREB) phosphorylation, followed by pCREB binding to a SOX-2 downstream enhancer [50]. These results all indicate a potential link between BPA exposure and cancer stem cell signaling pathways, contributing to increased cancer development and progression.

The bone morphogenetic protein (BMP) pathway might play a role in BPA-mediated breast cancer stem cell renewal and regulation as well [51]. BMP signaling is involved in the recruitment of stem cells in response to bone injury. However, this signaling pathway is also implicated in breast cancer malignancies [52]. BPA appears to increase the sensitivity of immature mammary epithelial cells (MCF-10A) to BMP signaling through receptor localization and downstream signal priming. Additionally, BPA exposure alters the fate of mammary stem cells by modulating their response to BMP2 and BMP4 [53].

\section{Epigenetic mechanisms of BPA action}

The vast majority of breast cancer cases are unrelated to germline mutations in tumor suppressor genes, and are, instead, thought to arise through either direct DNA damage, or epigenetic mechanisms, both of which can be induced by environmental stimuli. These cases of breast cancer are termed sporadic breast tumors [54]. BPA, BPF, and BPS have all been shown to induce estrogen-receptor mediated changes in the methylome of breast cancer cell lines and increased cell proliferation [55]. In recent years, it has been suggested that cancers induced through an endocrine disrupting epigenetic mechanism, such as BPA-induced, stem cell mediated breast cancers, can be prevented by bioactive food components [56].

Morgan et al. [57] found that BPA induced epigenetic changes in the coat color of viable yellow agouti mouse off-

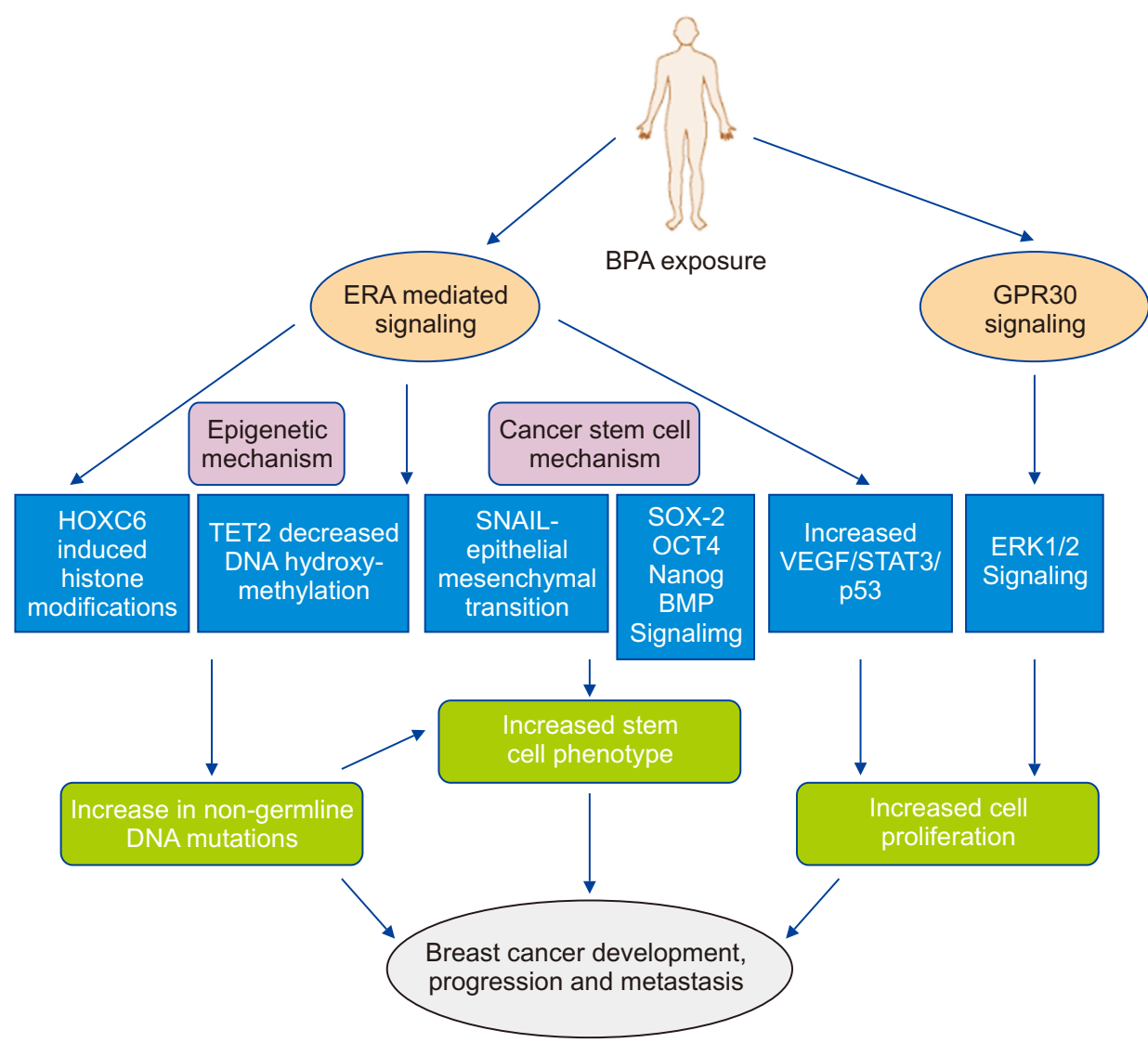

Figure 2. Potential mechanisms of BPA-induced breast cancer development and progression. BPA, bisphenol A; ERA, estrogen receptor alpha; GPR30, G-proteincoupled receptor 30; TET2, teneleven translocation 2; SNAIL, Snail family zinc finger protein; ERK1/2, extracellular signal-regulated kinase $1 / 2$. 
spring. This mouse model contains an intra-cisternal A particle retrotransposon which, when activated due to epigenetic modifications, leads to the expression of the agouti gene, of which the resulting protein causes a yellow coat color. This model can be used to map the effects of compounds on the epigenome [57]. The study by Dolinoy et al. [58] using this model found that the methyl-donors genistein and folate could reverse the hypomethylating effects of BPA, which caused a yellow coat color in viable yellow agouti mouse offspring.

A recent work by $\mathrm{Li}$ et al. [59] found that BPA-induced proliferation of MCF-7 cells was found to be mediated through suppression of DNA hydroxymethylation. Specifically, Ten-eleven translocation (TET)2 plays a direct role in DNA hydroxymethylation in response to BPA through interaction with ER $\alpha$ [59]. This novel pathway suggests a potential mechanism through which epigenetics play a role in breast cancer development in response to BPA exposure. Hussain et al. [60] have suggested the involvement of HOXC6, a homeobox-containing gene associated with breast cell development that is often found to be overexpressed in breast cancers. The estrogen response element in the promoter of the HOXC6 gene responds to BPA exposure, which facilitates the recruitment of ER $\alpha$ and estrogen receptor co-activators, ultimately leading to histone modifications and chromatin remodeling. Overexpression of HOXC6 also increased the expression of tumor growth factors in cell culture [60]. Another study noted the involvement of a second homeobox-containing gene, HOXB9. Through a similar mechanism, BPA was shown to induce expression of HOXB9 via ERs and ER co-activators, leading to the overexpression of HOXB9 and chromatin remodeling [61].

The long non-coding RNA HOX antisense intergenic RNA (HOTAIR), known to respond to estrogen to induce silencing of genes necessary for breast cancer proliferation, was found to respond to BPA through EREs and ER-coregulators in an MCF-7 model [62]. The study by Doherty et al. [63], modeled in CD-1 female mice, found that BPA induced the expression of EZH2, a histone methyltransferase involved in embryonic development, and often overexpressed during tumor development. Overall, these results highlight the importance of studying the potential epigenetic factors that influence the ability of BPA to induce carcinogenic effects. Figure 2 summarizes the possible signaling pathways/key molecules contributing to the increased breast cancer incidence and development following BPA exposure.

\section{CANCER PREVENTION PERSPECTIVE}

To establish better approaches to the prevention of breast cancer, it is important to recognize potential environmental determinants and to understand how they cause cancer development and progression. Industrialized society has ubiquitous environmental contaminants. Some are known to be harmful, but others have not been confirmed or even chal- lenged to assess for the long-term effects in humans.

Exposure to endocrine disruptors such as BPA has been suspected to increase the risk of breast cancer. Testing exposure and assessing cancer risks associated with chemicals via human trials is not ethical. However, using relevant epidemiological data, exposure assessment and toxicology, as well as well-defined mechanism studies altogether can build the best evidence needed for cancer prevention. Understanding the mechanistic link between BPA and cancer could help us to find a way to reduce breast cancer risk from the exposure as well as to further prevent breast cancer development. Clear elucidation of environmental causes in cancer development could provide effective long-lasting protection against breast cancer in humans.

\section{ACKNOWLEDGMENTS}

This research was supported by the National Institutes of Health grants, R01 AT007036, R01 CA127645, ES005022, New Jersey Health Foundation, Busch Biomedical Grant, and NIEHS/CEED Pilot Grant.

\section{CONFLICTS OF INTEREST}

No potential conflicts of interest were disclosed.

\section{ORCID}

Cassandra Winz, https://orcid.org/0000-0001-9993-9947

Nanjoo Suh, https://orcid.org/0000-0002-9962-9276

\section{REFERENCES}

1. Yager JD, Davidson NE. Estrogen carcinogenesis in breast cancer. N Engl J Med 2006;354:270-82.

2. Siegel RL, Miller KD, Fuchs HE, Jemal A. Cancer statistics, 2021. CA Cancer J Clin 2021;71:7-33.

3. Lakind JS, Naiman DQ. Bisphenol A (BPA) daily intakes in the United States: estimates from the 2003-2004 NHANES urinary BPA data. J Expo Sci Environ Epidemiol 2008;18:608-15.

4. Crinnion WJ. The CDC fourth national report on human exposure to environmental chemicals: what it tells us about our toxic burden and how it assist environmental medicine physicians. Altern Med Rev 2010;15:101-9.

5. Calafat AM, Ye X, Wong LY, Reidy JA, Needham LL. Exposure of the U.S. population to bisphenol A and 4-tertiary-octylphenol: 2003-2004. Environ Health Perspect 2008;116:39-44.

6. LaKind JS, Naiman DQ. Temporal trends in bisphenol A exposure in the United States from 2003-2012 and factors associated with BPA exposure: spot samples and urine dilution complicate data interpretation. Environ Res 2015;142:84-95.

7. Bao W, Liu B, Rong S, Dai SY, Trasande L, Lehmler HJ. Association between bisphenol $A$ exposure and risk of all-cause and cause-specific mortality in US adults. JAMA Netw Open 
2020;3:e2011620.

8. Böckers M, Paul NW, Efferth T. Bisphenolic compounds alter gene expression in MCF-7 cells through interaction with estrogen receptor $\alpha$. Toxicol Appl Pharmacol 2020;399:115030.

9. Weber Lozada K, Keri RA. Bisphenol A increases mammary cancer risk in two distinct mouse models of breast cancer. Biol Reprod 2011;85:490-7.

10. Dhimolea E, Wadia PR, Murray TJ, Settles ML, Treitman JD, Sonnenschein C, et al. Prenatal exposure to BPA alters the epigenome of the rat mammary gland and increases the propensity to neoplastic development. PLoS One 2014;9:e99800.

11. Ma Z, Parris AB, Howard EW, Davis M, Cao X, Woods C, et al. In utero exposure to bisphenol A promotes mammary tumor risk in MMTV-erbB2 transgenic mice through the induction of ERerbB2 crosstalk. Int J Mol Sci 2020;21:3095.

12. Wadia PR, Vandenberg LN, Schaeberle CM, Rubin BS, Sonnenschein C, Soto AM. Perinatal bisphenol A exposure increases estrogen sensitivity of the mammary gland in diverse mouse strains. Environ Health Perspect 2007;115:592-8.

13. ANSES's Working Group on "Endocrine disruptors", ANSES's Expert Committee on "Chemicals covered by the REACh and CLP Regulations”, Pouzaud F, Thierry-Mieg M, Burga K, Vérines-Jouin $L$, et al. Concerns related to ED-mediated effects of Bisphenol A and their regulatory consideration. Mol Cell Endocrinol 2018;475:92-106.

14. Paulose T, Speroni L, Sonnenschein C, Soto AM. Estrogens in the wrong place at the wrong time: fetal BPA exposure and mammary cancer. Reprod Toxicol 2015;54:58-65.

15. Chen D, Kannan K, Tan H, Zheng Z, Feng YL, Wu Y, et al. Bisphenol analogues other than BPA: environmental occurrence, human exposure, and toxicity-a review. Environ Sci Technol 2016;50:5438-53.

16. Rochefort H. Endocrine disruptors (EDs) and hormonedependent cancers: correlation or causal relationship? C R Biol 2017;340:439-45.

17. Soto AM, Brisken C, Schaeberle C, Sonnenschein C. Does cancer start in the womb? Altered mammary gland development and predisposition to breast cancer due to in utero exposure to endocrine disruptors. J Mammary Gland Biol Neoplasia 2013;18:199-208.

18. Atlas E, Dimitrova V. Bisphenol S and Bisphenol A disrupt morphogenesis of MCF-12A human mammary epithelial cells. Sci Rep 2019;9:16005.

19. Vandenberg LN, Maffini MV, Schaeberle CM, Ucci AA, Sonnenschein C, Rubin BS, et al. Perinatal exposure to the xenoestrogen bisphenol-A induces mammary intraductal hyperplasias in adult CD-1 mice. Reprod Toxicol 2008;26:210-9.

20. Fernandez SV, Russo J. Estrogen and xenoestrogens in breast cancer. Toxicol Pathol 2010;38:110-22.

21. Staples CA, Dorn PB, Klecka GM, O'Block ST, Harris LR. A review of the environmental fate, effects, and exposures of bisphenol A. Chemosphere 1998;36:2149-73.

22. Vandenberg LN, Chahoud I, Heindel JJ, Padmanabhan V,
Paumgartten FJ, Schoenfelder G. Urinary, circulating, and tissue biomonitoring studies indicate widespread exposure to bisphenol A. Environ Health Perspect 2010;118:1055-70.

23. Maffini MV, Rubin BS, Sonnenschein C, Soto AM. Endocrine disruptors and reproductive health: the case of bisphenol-A. Mol Cell Endocrinol 2006;254-255:179-86.

24. Morgan M, Deoraj A, Felty Q, Roy D. Environmental estrogenlike endocrine disrupting chemicals and breast cancer. Mol Cell Endocrinol 2017;457:89-102.

25. Smith-Bindman R. Environmental causes of breast cancer and radiation from medical imaging: findings from the Institute of Medicine report. Arch Intern Med 2012;172:1023-7.

26. Calaf GM, Ponce-Cusi R, Aguayo F, Muñoz JP, Bleak TC. Endocrine disruptors from the environment affecting breast cancer. Oncol Lett 2020;20:19-32.

27. Vandenberg LN, Ehrlich S, Belcher SM, Ben-Jonathan N, Dolinoy DC, Hugo ER, et al. Low dose effects of bisphenol A. Endocr Disruptors (Austin) 2013;1:e26490.

28. Hafezi SA, Abdel-Rahman WM. The endocrine disruptor bisphenol A (BPA) exerts a wide range of effects in carcinogenesis and response to therapy. Curr Mol Pharmacol 2019;12:230-8.

29. Huang B, Luo N, Wu X, Xu Z, Wang X, Pan X. The modulatory role of low concentrations of bisphenol $A$ on tamoxifen-induced proliferation and apoptosis in breast cancer cells. Environ Sci Pollut Res Int 2019;26:2353-62.

30. Lapensee EW, Tuttle TR, Fox SR, Ben-Jonathan N. Bisphenol A at low nanomolar doses confers chemoresistance in estrogen receptor-alpha-positive and -negative breast cancer cells. Environ Health Perspect 2009;117:175-80.

31. Thomas P, Dong J. Binding and activation of the seventransmembrane estrogen receptor GPR30 by environmental estrogens: a potential novel mechanism of endocrine disruption. J Steroid Biochem Mol Biol 2006;102:175-9.

32. Pupo M, Pisano A, Lappano R, Santolla MF, De Francesco EM, Abonante $S$, et al. Bisphenol $A$ induces gene expression changes and proliferative effects through GPER in breast cancer cells and cancer-associated fibroblasts. Environ Health Perspect 2012;120:1177-82.

33. Sengupta S, Obiorah I, Maximov PY, Curpan R, Jordan VC. Molecular mechanism of action of bisphenol and bisphenol $A$ mediated by oestrogen receptor alpha in growth and apoptosis of breast cancer cells. Br J Pharmacol 2013;169:167-78.

34. Lee HS, Park EJ, Oh JH, Moon G, Hwang MS, Kim SY, et al. Bisphenol A exerts estrogenic effects by modulating CDK1/2 and p38 MAP kinase activity. Biosci Biotechnol Biochem 2014;78:1371-5.

35. Mlynarcikova A, Macho L, Fickova M. Bisphenol A alone or in combination with estradiol modulates cell cycle- and apoptosisrelated proteins and genes in MCF7 cells. Endocr Regul 2013;47:189-99.

36. Fernandez SV, Huang Y, Snider KE, Zhou Y, Pogash TJ, Russo J. Expression and DNA methylation changes in human breast epithelial cells after bisphenol A exposure. Int $\mathrm{J}$ Oncol 
2012;41:369-77.

37. Lloyd V, Morse M, Purakal B, Parker J, Benard P, Crone M, et al. Hormone-like effects of bisphenol A on p53 and estrogen receptor alpha in breast cancer cells. Biores Open Access 2019;8:169-84.

38. Buteau-Lozano H, Velasco G, Cristofari M, Balaguer P, PerrotApplanat M. Xenoestrogens modulate vascular endothelial growth factor secretion in breast cancer cells through an estrogen receptor-dependent mechanism. J Endocrinol 2008;196:399-412.

39. Applanat MP, Buteau-Lozano H, Herve MA, Corpet A. Vascular endothelial growth factor is a target gene for estrogen receptor and contributes to breast cancer progression. Adv Exp Med Biol 2008;617:437-44.

40. Yamamoto T, Matsuda T, Junicho A, Kishi H, Saatcioglu F, Muraguchi A. Cross-talk between signal transducer and activator of transcription 3 and estrogen receptor signaling. FEBS Lett 2000;486:143-8.

41. Zhang W, Fang $Y$, Shi X, Zhang M, Wang X, Tan Y. Effect of bisphenol A on the EGFR-STAT3 pathway in MCF-7 breast cancer cells. Mol Med Rep 2012;5:41-7.

42. Siersbæk R, Scabia V, Nagarajan S, Chernukhin I, Papachristou EK, Broome R, et al. IL6/STAT3 signaling hijacks estrogen receptor $\alpha$ enhancers to drive breast cancer metastasis. Cancer Cell 2020;38:412-23.e9.

43. Song $H$, Zhang $T$, Yang P, Li M, Yang Y, Wang Y, et al. Low doses of bisphenol A stimulate the proliferation of breast cancer cells via ERK1/2/ERR $\gamma$ signals. Toxicol In Vitro 2015;30(1 Pt B):521-8.

44. Dong S, Terasaka S, Kiyama R. Bisphenol A induces a rapid activation of Erk1/2 through GPR30 in human breast cancer cells. Environ Pollut 2011;159:212-8.

45. Yu Z, Pestell TG, Lisanti MP, Pestell RG. Cancer stem cells. Int J Biochem Cell Biol 2012;44:2144-51.

46. Liu S, Wicha MS. Targeting breast cancer stem cells. J Clin Oncol 2010;28:4006-12.

47. Rodda DJ, Chew JL, Lim LH, Loh YH, Wang B, Ng HH, et al. Transcriptional regulation of nanog by OCT4 and SOX2. J Biol Chem 2005;280:24731-7.

48. Giovannelli P, Di Donato M, Galasso G, Di Zazzo E, Medici N, Bilancio A, et al. Breast cancer stem cells: the role of sex steroid receptors. World J Stem Cells 2019;11:594-603.

49. Yang L, Luo L, Ji W, Gong C, Wu D, Huang H, et al. Effect of low dose bisphenol A on the early differentiation of human embryonic stem cells into mammary epithelial cells. Toxicol Lett 2013;218:187-93.

50. Lillo MA, Nichols C, Seagroves TN, Miranda-Carboni GA, Krum $\mathrm{SA}$. Bisphenol A induces Sox2 in $\mathrm{ER}^{+}$breast cancer stem-like cells. Horm Cancer 2017;8:90-9.

51. Jung N, Maguer-Satta V, Guyot B. Early Steps of mammary stem cell transformation by exogenous signals; effects of bisphenol endocrine disrupting chemicals and bone morphogenetic proteins. Cancers (Basel) 2019;11:1351.

52. Chi LH, Burrows AD, Anderson RL. Bone morphogenetic protein signaling in breast cancer progression. Growth Factors 2019;37:12-28.

53. Clément F, Xu X, Donini CF, Clément A, Omarjee S, Delay E, et al. Long-term exposure to bisphenol $A$ or benzo(a)pyrene alters the fate of human mammary epithelial stem cells in response to BMP2 and BMP4, by pre-activating BMP signaling. Cell Death Differ 2017;24:155-66.

54. Kenemans P, Verstraeten RA, Verheijen RH. Oncogenic pathways in hereditary and sporadic breast cancer. Maturitas 2004;49:34-43.

55. Awada Z, Nasr R, Akika R, Cahais V, Cuenin C, Zhivagui M, et al. DNA methylome-wide alterations associated with estrogen receptor-dependent effects of bisphenols in breast cancer. Clin Epigenetics 2019;11:138.

56. Romagnolo DF, Daniels KD, Grunwald JT, Ramos SA, Propper $\mathrm{CR}$, Selmin OI. Epigenetics of breast cancer: modifying role of environmental and bioactive food compounds. Mol Nutr Food Res 2016;60:1310-29.

57. Morgan HD, Sutherland HG, Martin DI, Whitelaw E. Epigenetic inheritance at the agouti locus in the mouse. Nat Genet 1999;23:314-8.

58. Dolinoy DC, Huang D, Jirtle RL. Maternal nutrient supplementation counteracts bisphenol A-induced DNA hypomethylation in early development. Proc Natl Acad Sci USA 2007;104:13056-61.

59. Li Z, Lyu C, Ren Y, Wang H. Role of TET dioxygenases and DNA hydroxymethylation in bisphenols-stimulated proliferation of breast cancer cells. Environ Health Perspect 2020;128:27008.

60. Hussain I, Bhan A, Ansari KI, Deb P, Bobzean SA, Perrotti LI, et al. Bisphenol-A induces expression of HOXC6, an estrogenregulated homeobox-containing gene associated with breast cancer. Biochim Biophys Acta 2015;1849:697-708.

61. Deb P, Bhan A, Hussain I, Ansari KI, Bobzean SA, Pandita TK, et al. Endocrine disrupting chemical, bisphenol-A, induces breast cancer associated gene HOXB9 expression in vitro and in vivo. Gene 2016;590:234-43.

62. Bhan A, Hussain I, Ansari KI, Bobzean SA, Perrotti LI, Mandal SS. Bisphenol-A and diethylstilbestrol exposure induces the expression of breast cancer associated long noncoding RNA HOTAIR in vitro and in vivo. J Steroid Biochem Mol Biol 2014;141:160-70.

63. Doherty LF, Bromer JG, Zhou Y, Aldad TS, Taylor HS. In utero exposure to diethylstilbestrol (DES) or bisphenol-A (BPA) increases EZH2 expression in the mammary gland: an epigenetic mechanism linking endocrine disruptors to breast cancer. Horm Cancer 2010;1:146-55. 